Neuropsychological Dysfunction in Chronic Fatigue Syndrome

Accepted Manuscript: 27.02.2019

(C) 2019, American Psychological Association. This paper is not the copy of record and may not exactly replicate the final, authoritative version of the article. Please do not copy or cite without authors' permission. The final article will be available, upon publication, via its DOI: $10.1037 /$ neu0000550 
For new submissions, please be sure to include the submission checklist on the first page of your manuscript.

\author{
Neuropsychology ${ }^{\circledR}$ \\ Author(s) Agreement Checklist
}

As corresponding author, please be sure to use this form as a checklist to ensure proper submission of your manuscript. Failure to do so can result in the return of the manuscript without review.

Please check off the appropriate responses and submit it along with your cover letter. If you are not able to verify any of these, please describe the reasons in your cover letter.

I have read the Instructions to Authors contained on the Neuropsychology ${ }^{\circledR}$ website (www.apa.org/pubs/journals/neu/index.aspx).

I attest that all listed authors have contributed significantly to the manuscript and consent to their names on the manuscript.

I have prepared the manuscript in accordance with APA Publication Manual (6 $6^{\text {th }}$ ed.) guidelines.

I have included all elements of the manuscript (title page, abstract, body, tables, figures, and figure legends) within a single Rich Text Format (.rtf) or Word (.doc) file. PDF or any other format is not accepted.

I have included a structured abstract (objective, method, results, conclusions) with $\leq 250$ words and 3-5 key words.

I have provided a brief statement in the cover letter of how the article content is relevant to the domain of Neuropsychology as described in the journal inside cover.

I have included a cover letter that contains the names and contact information of all authors. Inclusion of such information indicates that these individuals have agreed to be an author.

I have read and agree to follow the APA Journals Internet Posting Guidelines (http://www.apa.org/pubs/authors/posting.aspx)

I attest that this manuscript is not being considered by another journal nor has it been published elsewhere. If applicable, I have provided information in my cover letter about any closely related manuscripts that have been submitted for consideration to the same or another journal. If the manuscript has been previously published in conference proceedings I have explained this in my cover letter.

I verify that if this manuscript describes a study wherein humans or animals were subjects, the treatment of those subjects was in accordance with established ethical guidelines and appropriate institutional approval has been obtained. The methods section indicates that the study received institutional ethics approval (including the name of the institution or committee) and that written informed consent was obtained from participants (where appropriate). 
$\nabla$ In describing the results of any statistical analyses, I have reported effect sizes and related confidence intervals where appropriate and relevant.

I have obtained permission to reproduce or adapt any copyrighted material from other sources and am able to provide documentation for this permission.

I have disclosed any information (in the Author Note or in my cover letter) about any interests or activities for myself or my coauthors that might be seen as influencing the research (e.g., financial interests in a test or procedure, funding by pharmaceutical companies for research).

I agree to comply with APA Ethics Code Standard 8.14a, Sharing Research Data for Verification, allowing other qualified professionals to confirm the analyses and results should my manuscript be accepted for publication. Per APA guidelines I will retain raw data for a minimum of 5 years after publication of the research.

$\checkmark$

If my manuscript is accepted for publication, I agree to transfer copyright to APA per the guidelines on the APA Publication Rights Form:

http//www.apa.org/pubs/authors/publication-rights-form.pdf

If masked review is requested, I have followed instructions to adequately conceal the identity of all authors to ensure masked review.

If my manuscript submission includes supplemental materials, I have explained the relevance of these materials in the cover letter, in the article itself, and within the supplemental materials. 


\section{Neuropsychological dysfunction in chronic fatigue syndrome and the relation between objective and subjective findings}

Omid Rasouli, ${ }^{1,2}$ Merethe Eide Gotaas, ${ }^{3,4}$ Ann-Katrin Stensdotter, ${ }^{1}$ Nils Inge Landr $\varnothing,{ }^{4,5}$ Pål Dåstøl, ${ }^{4,6}$ Eva Skovlund, ${ }^{8}$ Egil A. Fors ${ }^{7 *}$

${ }^{1}$ Department of Neuromedicine and Movement Science, Faculty of Medicine and Health Sciences, Norwegian University of Science and Technology, Trondheim, Norway

${ }^{2}$ Department of Mental Health, Faculty of Medicine and Health Sciences, Norwegian University of Science and Technology, Trondheim, Norway

${ }^{3}$ Department of Circulation and Medical Imaging, Faculty of Medicine and Health Sciences, Norwegian University of Science and Technology, Trondheim, Norway

${ }^{4}$ National Competence Center for Complex Symptom Disorders, St. Olav’s University Hospital, Trondheim, Norway

${ }^{5}$ Clinical Neuroscience Research Group, Department of Psychology, University of Oslo, Norway

${ }^{6}$ Division of Mental Health and Addiction, Vestre Viken Hospital Trust, Drammen, Norway

${ }^{7}$ Department of Public Health and Nursing, General Practice Research Unit, Faculty of Medicine and Health Sciences, Norwegian University of Science and Technology, Trondheim, Norway

${ }^{8}$ Department of Public Health and Nursing, Faculty of Medicine and Health Sciences, Norwegian University of Science and Technology, Trondheim, Norway

omid.rasouli@ntnu.no (phone: +47 94265117)

merethe.eide@ntnu.no (phone: +47 93002562)

ann-katrin.stensdotter@ntnu.no (phone: +46738092134)

n.i.landro@psykologi.uio.no (phone +47 95038479)

pal.dastol@vestreviken.no (phone: +47 48275263)

eva.skovlund@ntnu.no (phone: +47 73597536)

egil.a.fors@ntnu.no (phone +47 41236597)

*Corresponding author: Egil A. Fors, Department of Public Health and Nursing, General Practice Research Unit, Faculty of Medicine and Health Sciences, Norwegian University of Science and Technology, Trondheim, Norway. egil.a.fors@ntnu.no.

This study was funded by the Norwegian University of Science and Technology. The authors declare that they have no conflict of interest. 


\begin{abstract}
Objective: This study aimed to explore the relationship between self-reported cognitive difficulties, objective neuropsychological test performances, and subjective health complaints in chronic fatigue syndrome (CFS) and to examine the degree of impaired cognitive functions.

Method: A total of 236 consecutively recruited outpatients, aged between 18 and 62 years, completed the tests. Self-administered questionnaires were used for assessing fatigue, pain, depression, anxiety and subjective cognitive complaints (Everyday Memory Questionnaire, EMQ). Also, neuropsychological tests, i.e., Stroop I-IV, CVLT-II learning/delay, WAIS-III L-N Sequencing, and PASAT were performed to examine whether these objective measures correlated with subjective complaints and were compared with normative data. Results: There was a trend of association $(p<0.05)$ between unadjusted EMQ with Stroop IV (inhibition and shifting attention), CVLT-II learning/delay (verbal learning and memory) and WAIS-III L-N Sequencing (working memory), but none were statistically significant at the 0.001 level. EMQ was positively associated with fatigue, pain, and depression $(p<0.001)$. PASAT (working memory) was negatively associated with pain $(p<0.001)$. Between $21 \%$ and $38 \%$ of the patients performed below the 1.5 SD cut-off for clinically significant impairment on the Stroop tests. Conclusion: The self-reported cognitive performance was not strongly associated with the objective cognitive performances on any domains in patients with CFS. Patients with higher fatigue, pain and depression levels reported greater subjective cognitive difficulties, also higher pain related to lower objective working memory function. The CFS patients had problems mainly in domains of psychomotor speed and attention measured by the objective neuropsychological tests.
\end{abstract}

Keywords: neuropsychological tests; self-report; attention; depression; fatigue syndrome

Public Significance Statements: In patients with chronic fatigue syndrome, their self-reported cognitive performance reflects, to some extent, the objective cognitive performances on certain domains. They report a high level of cognitive difficulties, which are positively correlated with fatigue, pain, and depression; however, the relationships with objective tests are weak. They display problems mainly in domains of psychomotor speed and attentional control measured by objective neuropsychological performance tests. 
Chronic fatigue syndrome (CFS), sometimes referred to as myalgic encephalomyelitis (ME), is a severely devastating condition without any identifiable biomarkers. The diagnosis is made according to reported symptoms and criteria as defined by the Centers for Disease Control and Prevention criteria (Fukuda et al., 1994) or others, e.g., the Canadian criteria (Carruthers et al., 2003). Fatigue must be persistent, debilitating with a definite onset; and substantial fatigue is not dependent on ongoing exertion nor does it disappear after rest. The level of patient's daily activity must be reduced substantially and persistently over a minimum period of six months. Also, patients must have four or more additional symptoms; including post-exertional malaise, muscle pain, multi-joint pain without swelling or redness, newly onset headache, sleep disturbances, sore throat, tender lymph nodes, and cognitive dysfunction (Fukuda et al., 1994). Patients do not necessarily display all these mentioned symptoms and may experience fluctuations in symptom severity. Many patients undergo significant declines in their quality of life (Anderson \& Ferrans, 1997) and face significant functional disability (Anderson, Jason, Hlavaty, Porter, \& Cudia, 2012; Devendorf, Jackson, Sunnquist, \& Jason, 2017). Self-reported cognitive complaints are represented by attention or memory lapses, patients' selfperception (Baker, Gibson, Georgiou-Karistianis, \& Giummarra, 2018; Landrø et al., 2013) and everyday concerns with or without actual dysfunctions (Mitchell, 2008). Some studies have found no relationship between subjective complaints and performance on objective neuropsychological tests (Cockshell \& Mathias, 2014; Short, McCabe, \& Tooley, 2002;

Wearden \& Appleby, 1996). Other studies have in contrast observed a relationship between selfreported complaints and objective test performance, especially on impaired processing speed, attention, and memory (Claypoole et al., 2007; Cockshell \& Mathias, 2010; Constant et al., 2011; Dickson, Toft, \& O'Carroll, 2009; Friedberg, Dechene, McKenzie, \& Fontanetta, 2000; Jason et 
al., 1999; Majer et al., 2008; Schmaling \& Betterton, 2016; Van Den Eede et al., 2011). However, previous studies have some limitations; for example, some have not used validated diagnostic criteria or matched control groups, have not compared self-reported cognitive complaints and objective test performance directly or used aggregated data with specific cognitive domains (Cockshell \& Mathias, 2014; Ray, Phillips, \& Weir, 1993; Vercoulen et al., 1998). Heterogeneity in cognitive impairments and differences in symptom severity and comorbidities in individuals with CFS have been suggested as potential factors for inconsistencies findings across previous studies (Cvejic, Birch, \& Vollmer-Conna, 2016). Several factors, symptoms, and complaints are suggested to influence cognitive performances in CFS and contribute to the reported diversity subjective versus objective findings, such as fatigue, pain, depression, and anxiety (Capuron et al., 2006; Cockshell \& Mathias, 2014; Thomas \& Smith, 2009).

Some studies have revealed associations between fatigue and objective neuropsychological tests (e.g., memory and attention) (Joyce, Blumenthal, \& Wessely, 1996), whereas others have not (Capuron et al., 2006; Grafman et al., 1993; McDonald, Cope, \& David, 1993; Short et al., 2002; Vercoulen et al., 1998). Overall, fatigue (Teodoro, Edwards, \& Isaacs, 2018) and pain (Landrø et al., 2013; Moriarty, McGuire, \& Finn, 2011) seem to be related to impaired cognitive function. A recent study (Baker et al., 2018) has reported strong associations between the EMQ and objective tests of speed and complex attention in patients with chronic pain. There is no consensus whether depression and anxiety symptoms are associated with objective cognitive findings in CFS (Claypoole et al., 2007; Cockshell \& Mathias, 2014; DeLuca et al., 2004; Marshall, Forstot, Callies, Peterson, \& Schenck, 1997), but there are claims that depression (Christley, Duffy, Everall, \& Martin, 2013) and anxiety (Hughes, Hirsch, Chalder, \& Moss-Morris, 2016) are 
related to impaired cognitive function. However, in Functional Cognitive Disorder (FCD), a condition associated with impaired subjective cognitive symptoms, depression and anxiety are not regarded as the primary causes for these cognitive symptoms (Pennington, Hayre, Newson, \& Coulthard, 2015; Pennington, Newson, Hayre, \& Coulthard, 2015).

Moreover, neuropsychological difficulties are common in CFS and associated with impaired attention, memory and reaction time (Christley et al., 2013). According to previous studies (Santamarina-Perez, Eiroa-Orosa, Rodriguez-Urrutia, Qureshi, \& Alegre, 2014; Thomas \& Smith, 2009; Togo, Lange, Natelson, \& Quigley, 2015), the objectively measured neuropsychological findings for the CFS patients may be poorer than in the normal population. Accordingly, due to the above-mentioned inconsistent findings, it is relevant to tailor adequate neuropsychological test-batteries to cover the main cognitive functional areas of CFS. Therefore, the present study has three aims: to explore the association between subjective and objective assessments of cognitive neuropsychology measures, to explore the association between cognitive measures and subjective health complaints (fatigue, pain, depression, and anxiety), and to examine the degree of impairment objectively measured by neuropsychological findings compared to the normal population. Thus, our hypotheses were as follows: We hypothesized to find an incongruent subjective-objective relationship overall, but with positive associations for specific elements, e.g., attentional control and working memory. We also hypothesized that fatigue, pain, depression, and anxiety are related to impaired cognitive function and that the objectively measured neuropsychological findings for the CFS patients are poorer than in the normal population.

\section{Methods}




\section{Participants}

This study was part of a consecutive randomized controlled trial (Trial registration:

NCT00920777) with outcome assessed up to 52 weeks after baseline for eligible patients meeting the Fukuda/CDC 1994 criteria for CFS. A total of 626 patients with unspecified fatigue referred from their GP were assessed for eligibility at the CFS-clinic at St. Olav's Hospital in Trondheim [Norway] over a period of four years, and 246 outpatients with CFS, aged between 18 and 62 years, were recruited consecutively for participation in this study. Ten patients were later excluded due to missing data, and data from 236 patients were used for statistical analyses. All patients were diagnosed by a team consisting of a medical specialist (anesthesiology, psychiatry, general practice, and physical medicine and rehabilitation/PM\&R), a psychologist and a physiotherapist at the National Competence Center for Complex Symptom Disorders. The multidisciplinary team determined whether the patient fulfilled the CDC criteria for CFS (Fukuda et al., 1994) and thus implicitly had no other medical or psychological conditions, which could explain their symptoms. Exclusion criteria for all patients were neurological diseases, severe psychiatric disorders, or inflammatory rheumatologic diseases, non-fluency in the Norwegian language or pregnancy. Before entering the study, information was given and written informed consent was obtained from the patients. The study was approved by the Regional Ethical Committee (REK\#420082586) and conducted in accordance with the Declaration of Helsinki.

\section{Data acquisition}

Data acquisition comprised of self-report questionnaires and objective neuropsychological tests. All participants performed the tests in the same order, using standardized instructions and test 
procedures for each test (Strauss, Sherman, \& Spreen, 2006). The self-reported questionnaires and the neuropsychological tests were not obtained in the same session. The self-reported questionnaires were filled out in conjunction with the first assessment at the multidisciplinary fatigue clinic. The neuropsychological tests were performed two weeks after the first assessment at the fatigue clinic. The order of administration of the measures was the same for all the participants.

\section{Self-report questionnaires}

The patients completed the Norwegian Pain Association Minimum Questionnaire, NOSF-MIS (Fredheim et al., 2008), which is a test battery comprising EMQ, sociodemographic data, fatigue, pain, depression, and anxiety questionnaires.

\section{Cognitive complaints}

Cognitive complaints were obtained using the Everyday Memory Questionnaire (EMQ) (Sunderland, Harris, \& Gleave, 1984). It has 28 self-report items, and each item is rated on a 9point scale ranging from 1 (not at all in the last six months) to 9 (more than once a day). The mean value of 28 items (range 1-9) was used for further analysis, and higher scores reflect greater impairments. EMQ has a clear factor structure and good internal consistency (Cornish, 2000). Additionally, general memory and attentional function have been identified as the main factors in this questionnaire (Royle \& Lincoln, 2008).

\section{Fatigue}


The Chalder Fatigue Questionnaire (CFQ) was used to measure the severity of symptomatic fatigue rated by the participant (Chalder et al., 1993). The CFQ has 11 items based on Likert scores from 0-3, where a maximum score of 33 indicates utmost fatigue. Patients rated the symptom's frequency for each item by choosing one of four answers: "less than usual", "no more than usual", "more than usual", and "much more than usual". In accordance with recent studies, Likert scoring (0-33) was used instead of the bimodal scoring (range 0-11) (White et al., 2011).

\section{Pain}

The patients scored mean pain $(0-10)$, where $0=$ no pain, and $10=$ worst possible imagined pain, with mean, minimal and maximal scores during the last week using Brief Pain Inventory (BPI), (Cleeland \& Ryan, 1994). The average of these three values, (mean+ minimum+ maximum)/3, was used in the study. The published Norwegian translation of the BPI has shown good psychometric properties in a validation study (Klepstad et al., 2002).

\section{Depression}

The Beck depression inventory-II (BDI-II) was used to measure depressive symptoms (Beck, Steer, \& Brown, 1996). The BDI-II is a common subjective instrument to assess depression severity and has 21 self-rated items. The respondent should choose one of four statements, rankordered by severity according to how frequently they experienced each symptom during the past week. Each item is scored from 0 for a neutral statement (I do not feel disappointed in myself) to 3 for the most severe statement (I hate myself). The sum score of all items is considered as the depression score. Categorization into minimal (0-13), mild (14-19), moderate (20-29), and severe (30-63) was used as previously specified by Beck et al. (Beck et al., 1996). 
Anxiety

Anxiety was measured using the subscale of anxiety from the Hospital Anxiety and Depression Scale (HADS). This questionnaire has seven items for anxiety (HADS-A). Each item on the questionnaire is scored 0-3, and a person can score 0-21 for either anxiety or depression (Zigmond \& Snaith, 1983). A cutoff score of 11 is frequently used to identify clinical levels of anxiety in the general population. However, a lower cutoff of 9 has been recommended to be used in CFS participants when compared to gold-standard measures (Morriss \& Wearden, 1998).

\section{Objective neuropsychological tests}

A psychologist administered the objective neuropsychological testing. All tasks are well-known and validated tests implemented in standard procedures of clinical neuropsychological assessment procedures (Lezak, Howieson, Bigler, \& Tranel, 2012). We used well-established test

procedures with published normative data adjusted for age and years of education in this study to explore basic cognitive functions including memory, working memory, verbal learning, attention, and psychomotor speed as well as indicators of general intelligence (Strauss et al., 2006).

\section{General intelligence}

As measures of general intelligence, two subtests of Vocabulary and Matrix Reasoning in the Wechsler Adult Intelligence Scale-Third Edition (WAIS-III) were employed (Wechsler, 2003), which are highly correlated with verbal and nonverbal IQ, respectively. Vocabulary subset is a gauge of verbal comprehension and mostly reflects premorbid functioning. In this test, the 
patients explained the meaning of words with increasing difficulty. In the Matrix Reasoning test, the patients were presented with a series of increasingly difficult visual pattern completions and analogy problems. The patients had to choose the one that best completed the pattern among five different alternatives (Strauss et al., 2006). In both tests, higher standardized scores indicate better performance.

\section{Psychomotor speed and attention}

In the present study, the participants performed the version of the classic Stroop test from the Delis-Kaplan Executive Functions System, which has four conditions and is named the ColorWord Interference test (Delis, Kaplan, \& Kramer, 2001). The first condition was naming the color of 50 different patches (blue, green and red). In the second condition, patients read 50 color names (blue, green and red) printed in black ink. Stroop I and II measure psychomotor speed. The third condition was an interference condition where the patients had to name the color of the ink of 50 color words. The color of the ink was different from the written color word. Stroop III, thus to focus attention on the color, measures the ability to inhibit a dominant and automatic verbal response. The fourth condition adds a switching condition, that is, the rule was the same as the third condition, except when the word had a frame around it, and the patients had to read the word instead of naming the color of the ink. Stroop IV is, thus, a measure of both verbal inhibition and cognitive flexibility and ability to switch attention. In all four conditions, the patients were instructed to perform the task as quickly and correctly as possible. Thus, higher standardized scores indicate better performance.

\section{Working memory}


Working memory was measured by two different tests including Letter Number (L-N)

Sequencing from the WAIS-III (Wechsler, 2003) and Paced Auditory Serial Addition Task

(PASAT). WAIS-III L-N Sequencing Test determined the ability to keep a specific amount of information for a limited period (i.e., storage capacity) (Wechsler, 2003). PASAT measured the ability to continuously update and effectively hold information in working memory over short time intervals; it also has a time element (Strauss et al., 2006). In the WAIS-III L-N Sequencing Test, the patients had to record sets of numbers and letters. The patients were read a series of numbers and letters combined randomly, and the length of the series was gradually increased. The patient had to remember the series and reorganize them with numbers in ascending order followed by letters in alphabetical order. Points were given for each correct item, and this score was age-corrected, and the higher (raw and standard) scores the better. In PASAT, the patients listened to an audiotape in which 61 digits were read successively with a 2-second inter-stimulus interval (Landrø, Celius, \& Sletvold, 2004). Patients were instructed to add each 1-digit number presented to the digit immediately preceding it and verbally tell the sum to the administrator. The number of correct calculations was marked as the score and then converted to standardized scores. Higher mean standardized scores show better performance. If anyone aborted the test before reaching 20 digits, the score was regarded as a missing value.

\section{Verbal learning and memory}

The California Verbal Learning Test-Second Edition (CVLT-II) assessed verbal learning and various aspects of memory (Delis, Kaplan, Kramer, \& Ober, 2000). The CVLT-II reveals different strategies, processes, and errors an individual may show during a learning task (Delis, Freeland, Kramer, \& Kaplan, 1988). The test was an oral presentation of a list of 16 words over 
five trials. There were four different categories, and each of the 16 words belonged to one of them. The patients were instructed to repeat all the words they could remember after each trial (immediate recall). The verbal learning score was the sum of all words remembered over the five trials. The CVLT-II delay score was calculated as the sum of remembered words after 20 minutes.

\section{Detection of inadequate effort}

The CVLT-II Forced Choice was used to assess and ensure the validity of the participant's responses (Denning, 2012). The Norwegian Version of CVLT-II forced-choice recognition followed the procedure described in the test manual (Siqveland, Sundseth, Dalsbo, Harboe, \& Leiknes, 2014). Forced choice recognition was the last task in the CVLT-II test battery. After the above-described test of immediate recall of 16 words from four categories (list A), the patient was given a new list of 16 words (list B) to recall once and then forget. The following instruction was to recall the words from list A (short delay interval). This test was followed by cued recognition with instructions to recall items from each of the four named categories. After a 30minute break, the patient was asked to recall the words from list A (long delay interval) followed by a second cued recognition by category. The patient was then presented for a list of 48 words which included the 16 words from list A and asked to answer yes or no to whether each word presented was part of list A (yes/no recognition). After a five-minute break the patient was presented for 16 pairs of words and asked to identify the word from list A in each pair (forced choice recognition). Higher standardized scores represent better results.

\section{Statistical analyses}


Statistical Package for the Social Sciences (SPSS, Version 25, IBM Corporation, USA) was used to analyze the data. The data were tested for normality with the Shapiro-Wilk test, histograms and residual plots for visual inspection. The associations between the standardized scores of the objective cognitive tests and self-reported cognitive complaints (EMQ) were explored using simple and multiple linear regression analysis, with and without adjusting for fatigue, pain, depression, and anxiety. Assumptions were checked by visual inspection of residual plots. The associations between pain, fatigue, depression, and anxiety with objective and subjective cognitive tests were explored using bivariate correlation analysis. The data of the participants were compared to the published normative data to assess functional impairments. The standardized scores were used when the objective test performances were compared to normative data. The percentage of patients performing 1.5 standard deviations (SD) and 2.0 SDs below the normative mean, respectively, were tested with z-tests. Due to the number of multiple comparisons and to reduce the risk of false-positive results, a nominal significance level of 0.001 was chosen.

\section{Results}

Table 1 shows the patients' characteristics. The sample was predominantly female (82.2\%) with a mean age of 35.7 years, and $31.8 \%$ had a university education. Overall, level of fatigue was moderately high (CFS score mean of 25.5 SD 4.8), pain levels were low to moderate (BPI score mean of 4.3), and they mostly did not report clinically significant anxiety or depressive symptoms. 
The data were normally distributed. However, the CVLT-II delayed recall variable was rather skewed, but regarded as normally distributed because of the large sample size $(n=236)$, and it was included in the multiple regression models as an independent variable.

Table 1 in here

\section{Associations between subjective and objective cognitive tests}

Table 2 lists the results of multiple regression analysis assessing associations between objective neuropsychological tests and self-reported cognitive function (EMQ), with and without adjusting for fatigue, pain, depression, and anxiety. The standardized coefficients (Beta / $\beta$ ) were relatively similar between the unadjusted and adjusted model (taking into account fatigue, pain, depression, and anxiety variables). There was also a very small model adjusted $\mathrm{R}^{2}$ for both unadjusted and adjusted EMQ ranging from 0.001 to 0.040 . The unadjusted EMQ was weakly and negatively associated with Stroop IV, CVLT-II learning, CVLT-II delay, and WAIS-III L-N sequencing; however, the small coefficients $(\beta<-0.17, p<0.05)$ and did not reach $p<0.001$. Adjusted EMQ for fatigue, pain, depression, and anxiety were also negatively and weakly associated with CVLT-II learning and CVLT-II delay $(\beta<-0.17, p<0.05)$, but again did not reach $p<0.001$. This indicates a weak trend where increasing subjective cognitive complaints are associated with poorer objective neuropsychological performances for some tests. In the CVLT-II Forced Choice embedded validity test, 232 subjects out of 236 (98\%) scored 16/16 correct raw scores, two scored 15/16 correct raw scores, while two were missing.

Table 2 in here 
Fatigue, pain, anxiety, depression related to cognitive measurements

The EMQ score was positively associated with fatigue (CI: $0.233,0.476, p<0.001)$, pain (CI:

$0.128,0.367, p<0.001)$ and depression (CI: $0.163,0.396, p<0.001$ ), but not with anxiety (Table

3). For objective cognitive measures, there was only one significant correlation between PASAT and pain (CI: $-0.344,-0.124, p<0.001)$ indicating that higher pain was associated with poorer working memory (Table 3).

Table 3 in here

Impaired cognitive functioning

Table 4 displays the percentage of patients performing worse than 1.5 and 2.0 SDs below the normative mean on objective cognitive measures. Stroop I-IV varied between 21-38\% below the norm in patients performing worse than $1.5 \mathrm{SD}$, with Stroop I displaying speed as the most impaired factor. Only a small number of the CFS patients displayed impairments on the other objective neuropsychological tests.

Table 4 in here

\section{Discussion}

A total of 236 CFS patients consecutively recruited in tertiary care were examined with selfreported questionnaires and neuropsychological tests. There was a trend of weak associations between self-reported complaints of cognitive impairments (EMQ) with some objective 
cognitive deficits regarding basic aspects of attentional control (shifting attention and inhibition), verbal learning/memory, and working memory. However, none of the associations were statistically significant at the 0.001 level. EMQ correlated with fatigue, pain and depression, but not with anxiety. Also, pain as the only health complaint, correlated with one objective cognitive test, i.e., PASAT, indicating worsening working memory with an increasing pain level. The CFS patients presented reduced psychomotor speed and attentional control compared to the norm, that is, up to $38 \%$ scored $1.5 \mathrm{SD}$ below the normative mean measured by objective neuropsychological tests.

\section{The relations between subjective and objective cognitive findings}

Our results showed a trend of weak associations between subjective and some of the objective cognitive tests, even after controlling for the levels of fatigue, pain, depression, and anxiety. Thus, our results support some previous studies that show discrepancies between subjective and objective cognitive measures in CFS (Cockshell \& Mathias, 2014; Cvejic, Lloyd, \& VollmerConna, 2016; Krupp, Sliwinski, Masur, Friedberg, \& Coyle, 1994; Short et al., 2002; Teodoro et al., 2018). Several explanations for these discrepancies have been proposed by different authors. Cockshell \& Mathias (2013) and others (Vercoulen et al., 1998; Wearden \& Appleby, 1996) have suggested that patients with CFS could overestimate their cognitive problems in their subjective reports rather than having real, objective memory deficits. Other possible explanation about these discrepancies could be the possibility that the cognitive tests are not sufficiently sensitive to study the cognitive problems of these patients, due to the low ecological validity of some tests, and due to low effort strategies applied by these patients because mental effort is aversive for them (Wearden \& Appleby, 1996). Others have suggested that they overestimate 
their premorbid cognitive performance, like the "good-old-days" phenomenon (Sullivan \& Edmed, 2012). The results also indicated that CFS might mimic functional cognitive disorder (FCD), i.e., a condition which has been suggested to have a subjective experience of cognitive dysfunction in the absence of underlying brain pathology (Pennington, Hayre, et al., 2015; Teodoro et al., 2018).

It has been suggested that memory complaints in patients with CFS may be due to deficits in information processing speed rather than actual memory deficits (Cockshell \& Mathias, 2013) and it is probably one of the most common features of the condition (Michiels \& Cluydts, 2001). Not all studies have found subjective versus objective test discrepancies, but rather noteworthy positive relationships. Some studies have revealed an impaired cognitive function in the objectively measured memory domains (Cockshell \& Mathias, 2010, 2013; Ickmans et al., 2013; Majer et al., 2008). However, as these also found reduced (Cockshell \& Mathias, 2013; Ickmans et al., 2013) or inexplicit subjective measures (Cockshell \& Mathias, 2010; Majer et al., 2008), they did not support our findings concerning no relationships between subjective and objective cognitive outcomes (see Table 2).

A few factors may explain the inconsistencies between the studies including wide variation in sample characteristics (size and heterogeneity), and substantial variation in the cognitive tests (Goedendorp, van der Werf, Bleijenberg, Tummers, \& Knoop, 2013; Ojeda, Failde, Duenas, Salazar, \& Eccleston, 2016). For example, some studies used standardized neuropsychological tests, whereas others employed computerized experimental tasks (Baker et al., 2018). Selection of the self-report questionnaires on cognitive complaints is an important factor which could be a potential reason for inconsistencies in the previous findings (Cvejic, Lloyd, et al., 2016). Various kinds of such questionnaires have been used in different studies, for example, 25-item Cognitive 
Failures Questionnaire and the 18-item Everyday Attention Questionnaire (Cockshell \& Mathias, 2014), 34-item Somatic and Psychological Health Report (SPHERE) (Cvejic, Lloyd, et al., 2016), The Subjective Mental Abilities Rating Test (SMART) (Short et al., 2002), and Cognitive Failures Questionnaire (CFQ) (Wearden \& Appleby, 1997). Our motivation for choosing EMQ was that the 28-item EMQ defines five clear factors: retrieval, task monitoring, conversational monitoring, spatial memory, and memory for activities, which reflect underlying memory processes (Cornish, 2000). Cornish has suggested that the EMQ is a psychometrically sound test, with good internal consistency and simplicity of administration (Cornish, 2000).

Another explanation for a subjective-objective test incongruence could be different contents between the subjective and objective tests, that is, that they measure different constructs or domains in the EMQ and the objective neuropsychological test battery. This subjective cognitive questionnaire (EMQ) is known primarily to assess memory and attention (Sunderland et al., 1984), while the objective cognitive test battery is generally comprehensive and contains psychomotor speed, attentional control, and verbal learning /memory in addition to working memory. Hence, our results support the view that EMQ primarily measures attention and memory because in our study it showed a trend towards correlation with the objective tests for aspects of attention and memory and less with speed, which means that the contents of the subjective cognitive complaints and objective tests appeared dissimilar. Therefore, it may not only matter whether the subjective and cognitive tests correlate, but for which domains they do so. That findings of deficits in measured psychomotor speed did not correlate with EMQ suggests that EMQ probably does not test this domain. Some authors (Cockshell \& Mathias, 2010, 2013; Ickmans et al., 2013; Majer et al., 2008) who found impaired cognitive performance in CFS (in contrast to, e.g., Cockshell \& Mathias 2014), concluded that speed is most often 
reduced, while deficits in memory and attention domains are more infrequent (Cockshell \& Mathias, 2013). Similar to patients with CFS, studies on chronic pain patients have reported associations between outcomes in subjective assessment of cognition and poorer attention and executive function performances (Baker et al., 2018; Landrø et al., 2013; Tesio et al., 2015). Baker and colleagues (2018) have reported strong associations between EMQ and objective tests such as speed and complex attention in patients with chronic pain and recommend that the EMQ may be a suitable indicator of cognitive difficulty in persons with pain since it is quick to administer (Baker et al., 2018).

\section{Association between cognitive measures and subjective health complaints}

Fatigue, pain, and depression correlated positively with EMQ showing that CFS patients report more cognitive impairments with higher levels of fatigue, pain and depression. The positive relation between EMQ and fatigue was in line with Aasvik and colleagues (2015), who also found a strong association between fatigue and EMQ among patients on sick leave, and proposed that individuals with fatigue may perceive impaired memory function because of their difficulty in accessing cognitive resources to perform different everyday tasks (Aasvik et al., 2015). Thus, reducing the fatigue level may help to reduce subjective complaints in patients with CFS. Our results also supported studies that did not find any associations between perceived fatigue and objective neuropsychological tests (Grafman et al., 1993; McDonald et al., 1993; Short et al., 2002; Vercoulen et al., 1998) and in contrast to others, which have found disruptions in the attention domain with increased perceived fatigue (Capuron et al., 2006; Joyce et al., 1996). Subjective memory complaints and pain are both considered to be important features of CFS (Fukuda et al., 1994), and patients with chronic pain often complain about their poor memory 
(Mazza, Frot, \& Rey, 2018). Thus, our results that higher pain level was associated with poorer subjective memory was in line with previous studies in patients with chronic pain, for example fibromyalgia (Elkana, Falcofsky, Shorer, Bar-On Kalfon, \& Ablin, 2019).

Further, similar to us, Cockshell \& Mathias (2014) found that subjective memory and attention complaints were associated with depressive symptoms, while objective cognitive measures were not, which are in contrast to another study where depression and objective cognitive tests correlated (Christopher \& MacDonald, 2005). It is believed that psychological dysfunction causes impairment in working memory, attention, and other cognitive skills (Airaksinen, Larsson, \& Forsell, 2005; Castaneda, Tuulio-Henriksson, Marttunen, Suvisaari, \& Lonnqvist, 2008). Airaksinen et al. (2005) have found impairments in both episodic memory and executive functioning, but not verbal fluency and psychomotor speed, in individuals diagnosed with anxiety disorders (i.e., panic disorder, generalized anxiety disorder, and obsessive-compulsive disorder). However, in our study, we found no associations between psychological dysfunction (i.e., depression and anxiety) and objective cognitive tests. In general, the contradictory findings may be results of methodological differences in the previous studies regarding the selection of participants, patient status, and objective cognitive tests (Airaksinen et al., 2005), e.g. the patients in our study scored low on anxiety and depression.

Although there were no associations between objective cognitive measures and subjective health complaints in our study, pain correlated with PASAT (working memory including a time element and a mental arithmetic task) as the only objective cognitive measure, indicating that higher levels of pain in CFS patients would adversely affect working memory performance. The integrity of cognitive processes has been associated with the ability to regulate responses to pain in both chronic and experimental pain conditions (Bjekic, Zivanovic, Puric, Oosterman, \& 
Filipovic, 2018; Karsdorp, Geenen, \& Vlaeyen, 2014). However, studies regarding associations between pain and cognitive functions are generally contradictory. For instance, Ickmans et al. (2013) did not find any correlation between pain and objective cognitive performance in CFS (Ickmans et al., 2013), but reported significant associations between pain and psychomotor response speed in a later study (Ickmans et al., 2015). In a study by Landrø et al. (2013), the Stroop I-IV scores in patients with generalized pain were impaired almost similar to the scores in our current study. It appears, thus, that attention and psychomotor speed (the Stroops) in CFS are affected similarly to that in the patients with generalized pain/fibromyalgia. These similarities may be explained by chronification affecting the same regulatory neural mechanisms in CFS as well as in pain conditions (Ingvar, 2015). In our study, the high numbers of correct raw scores of the CVLT-II Forced Choice asserted that appropriate effort and task engagement had occurred.

\section{Impaired Cognitive Functioning}

More than a third of the patients performed below the cut-off for a clinically significant neuropsychological impairment (1.5 SD) in the Stroop tests, with Stroop I (38\%) and II (31\%) as the most impaired. It suggests that reduced psychomotor speed is a major neuropsychological problem for CFS patients, but also attentional control (Stroop III and IV). Our findings are in accordance with other neuropsychological studies on CFS, which have reported slower psychomotor speed (Cockshell \& Mathias, 2013) in addition to impaired attention in Stroop tests (Santamarina-Perez et al., 2014; Thomas \& Smith, 2009; Togo et al., 2015). However, our results revealed that only minor numbers of patients had impairments in other cognitive tests such as working memory, and verbal learning and delayed memory (see Table 4), in contrast to, for example, Cockshell's meta-analysis (Cockshell \& Mathias, 2010) and others (Majer et al., 
2008). Although memory problem is one of the main subjective complaints in patients with CFS, our findings, and previous studies have shown that memory function is nearly intact according to most objective neuropsychological tests on performance (Santamarina-Perez et al., 2014). Shank et al. (2013) have suggested that patients with CFS do not have problems with memory per se, but rather with the processing of new and/or complex information, particularly when information is presented quickly (Shanks, Jason, Evans, \& Brown, 2013). General dysfunction in attention and information processing speed have been suggested as reasons for complaints of memory problems in CFS (DeLuca et al., 2004; Dickson et al., 2009).

In the present study, we used 1.5 SD as the main cut-off threshold for neuropsychological impairment, but also report the percentages that performed below the $2.0 \mathrm{SD}$ cut-off limit for the normative data as extra information for comparison with other studies (see Table 4). Although there are no absolute rules regarding criteria for neuropsychological impairment and no consensus regarding the cut-off level, we consider $1.5 \mathrm{SD}$ as most adequate. If a less strict cutoff of, say, $1 \mathrm{SD}$ had been selected, there would have been a risk for healthy persons to be regarded as impaired (false positive), and with the stricter 2.0 SD cut-off, impaired patients could have been regarded as healthy (false negative) (Landrø et al., 2013). Nevertheless, Majer et al. (2008) claimed that raising the cutoff from 1.0 to $1.5 \mathrm{SD}$ would yield only modest gains in specificity, but with a "large trade-off" in its sensitivity. Normative data for each cognitive domain are based on large samples of age- and gender-matched subjects (Strauss et al., 2006).

Some limitations of the present study should be considered for interpretation and implication of the findings. As mentioned above, we used normative data to compare the findings on the neuropsychological tests to expected normal test performance; however, not including a healthy control group may be considered as a limitation since participants' characteristics of the 
experimental group may be different from the norm group. Another limitation to consider is that we did not include the years of CFS evolution, though, all the patients had illness duration longer than six months, as diagnosed according to the CDC (Fukuda et al., 1994). Moreover, sleep, alcohol use, medicine, and nicotine use were not specified or controlled for, which may potentially have affected performance, and need to be investigated in future studies. However, very few used daily medication, and nobody had alcohol issues, as iatrogenic conditions such as side effects of medications and alcohol problems are excluding factors from the CFS diagnosis. External validity is limited as these patients were handled in tertiary care; the generalization of results in primary care must, therefore, be made with caution. Findings should furthermore be interpreted with caution, as this study had an explorative character.

The strengths of the study were a large sample size, consecutive enrollment procedure, the use of a multidisciplinary and highly competent diagnostic team plus a well-founded and selective subjective and objective neuropsychological test battery. Further research will need to develop reliable self-report questionnaires on cognitive complaints to capture the cognitive difficulties in a broader sense than the EMQ and take processing speed and attentional control into account. As it appears now, EMQ alone is not sufficient and must be supplemented, for example, by the Stroop I-IV tests and embedded measures of performance validity, e.g., the CVLT-II Forced Choice, to provide a comprehensive, valid, and adequate test battery for patients with CFS. It is reasonable to develop a brief and reliable neurocognitive battery specifically tailored and standardized for individuals with CFS, which can be employed both in routine clinical practice and as a research and evaluation tool. Helping patients in reducing the level of depression, pain and fatigue may result in reducing subjective cognitive complaints and higher confidence in cognitive functioning. Moreover, pain reduction may improve working memory as shown by the 
negative association between pain and PASAT. It is important for practitioners to consider the heterogeneity in CFS patients due to different number and severity of symptoms when the patients are treated, and the main focus should be directed to the attention and psychomotor speed dysfunction in these patients.

\section{Conclusions}

Self-reported cognitive impairments did not correlate significantly with the objective neuropsychological tests, albeit there were trends of weak associations for attentional control, verbal learning /memory and working memory in this large group of consecutively recruited patients with CFS. Patients with higher fatigue, pain and depression levels reported more subjective cognitive difficulties, but did not generally display cognitive dysfunction in objective tests; with the exception that pain affected working memory in a negative way. A relatively high percentage of the patients performed below the limit for normal functioning in some cognitive domains measured by objective neuropsychological tests, particularly psychomotor speed and attentional control. This pattern of findings will influence functional abilities in daily life. Our results indicate that cognitive functioning should be systematically included in the routine evaluation of patients with CFS, and a combination of a subjective self-report questionnaire with formal, objective assessment seems optimal.

\section{Acknowledgments}

We gratefully thank all the participants and the National Competence Center for Complex Symptom Disorders. Our sincere appreciation goes to Inger Storaker, Ann-Elise Solvang and Nina Groven for administering the patients, and to Nora Nilssen for preparing the dataset from 
the questionnaires. This study was funded by the Liaison Committee for Education, Research and Innovation in Central Norway. 


\section{References}

Aasvik, J. K., Woodhouse, A., Jacobsen, H. B., Borchgrevink, P. C., Stiles, T. C., \& Landrø, N. I. (2015). Subjective memory complaints among patients on sick leave are associated with symptoms of fatigue and anxiety. Frontiers in Psychology, 6, 1338. doi:10.3389/fpsyg.2015.01338

Airaksinen, E., Larsson, M., \& Forsell, Y. (2005). Neuropsychological functions in anxiety disorders in population-based samples: evidence of episodic memory dysfunction. Journal of Psychiatric Research, 39(2), 207-214. doi:10.1016/j.jpsychires.2004.06.001

Anderson, J. S., \& Ferrans, C. E. (1997). The quality of life of persons with chronic fatigue syndrome. Journal of Nervous and Mental Disease, 185(6), 359-367.

Anderson, V. R., Jason, L. A., Hlavaty, L. E., Porter, N., \& Cudia, J. (2012). A review and meta-synthesis of qualitative studies on myalgic encephalomyelitis/chronic fatigue syndrome. Patient Education and Counseling, 86(2), 147-155. doi:10.1016/j.pec.2011.04.016

Baker, K. S., Gibson, S. J., Georgiou-Karistianis, N., \& Giummarra, M. J. (2018). Relationship between selfreported cognitive difficulties, objective neuropsychological test performance and psychological distress in chronic pain. European Journal of Pain (London, England), 22(3), 601-613. doi:10.1002/ejp.1151

Beck, A. T., Steer, R. A., \& Brown, G. K. (1996). Manual for the beck depression inventory (BDI-II). In (2nd ed. ed.). San Antonio, TX: Psychological Corporation.

Bjekic, J., Zivanovic, M., Puric, D., Oosterman, J. M., \& Filipovic, S. R. (2018). Pain and executive functions: a unique relationship between Stroop task and experimentally induced pain. Psychological Research, 82(3), 580-589. doi:10.1007/s00426-016-0838-2

Capuron, L., Welberg, L., Heim, C., Wagner, D., Solomon, L., Papanicolaou, D. A., .. . Reeves, W. C. (2006). Cognitive dysfunction relates to subjective report of mental fatigue in patients with chronic fatigue syndrome. Neuropsychopharmacology, 31(8), 1777-1784. doi:10.1038/sj.npp.1301005

Carruthers, B. M., Jain, A. K., De Meirleir, K. L., Peterson, D. L., Klimas, N. G., Lerner, A. M., . . van de Sande, M. I. (2003). Myalgic encephalomyelitis/chronic fatigue syndrome: clinical working case definition, diagnostic and treatment protocols. Journal of chronic fatigue syndrome, 11(1), 7115.

Castaneda, A. E., Tuulio-Henriksson, A., Marttunen, M., Suvisaari, J., \& Lonnqvist, J. (2008). A review on cognitive impairments in depressive and anxiety disorders with a focus on young adults. Journal of Affective Disorders, 106(1-2), 1-27. doi:10.1016/j.jad.2007.06.006

Chalder, T., Berelowitz, G., Pawlikowska, T., Watts, L., Wessely, S., Wright, D., \& Wallace, E. P. (1993). DEVELOPMENT OF A FATIGUE SCALE. Journal of Psychosomatic Research, 37(2), 147-153. doi:10.1016/0022-3999(93)90081-p

Christley, Y., Duffy, T., Everall, I. P., \& Martin, C. R. (2013). The neuropsychiatric and neuropsychological features of chronic fatigue syndrome: revisiting the enigma. Curr Psychiatry Rep, 15(4), 353. doi:10.1007/s11920-013-0353-8

Christopher, G., \& MacDonald, J. (2005). The impact of clinical depression on working memory. Cognitive Neuropsychiatry, 10(5), 379-399. doi:10.1080/13546800444000128

Claypoole, K. H., Noonan, C., Mahurin, R. K., Goldberg, J., Erickson, T., \& Buchwald, D. (2007). A twin study of cognitive function in chronic fatigue syndrome: the effects of sudden illness onset. Neuropsychology, 21(4), 507-513. doi:10.1037/0894-4105.21.4.507

Cleeland, C. S., \& Ryan, K. M. (1994). Pain assessment: Global use of the brief pain inventory. Annals Academy of Medicine Singapore, 23(2), 129-138. 
Cockshell, S. J., \& Mathias, J. L. (2010). Cognitive functioning in chronic fatigue syndrome: a metaanalysis. Psychological Medicine, 40(8), 1253-1267. doi:10.1017/S0033291709992054

Cockshell, S. J., \& Mathias, J. L. (2013). Cognitive deficits in chronic fatigue syndrome and their relationship to psychological status, symptomatology, and everyday functioning. Neuropsychology, 27(2), 230-242. doi:10.1037/a0032084

Cockshell, S. J., \& Mathias, J. L. (2014). Cognitive functioning in people with chronic fatigue syndrome: a comparison between subjective and objective measures. Neuropsychology, 28(3), 394-405. doi:10.1037/neu0000025

Constant, E. L., Adam, S., Gillain, B., Lambert, M., Masquelier, E., \& Seron, X. (2011). Cognitive deficits in patients with chronic fatigue syndrome compared to those with major depressive disorder and healthy controls. Clinical Neurology and Neurosurgery, 113(4), 295-302.

doi:10.1016/j.clineuro.2010.12.002

Cornish, I. M. (2000). Factor structure of the everyday memory questionnaire. British Journal of Psychology, 91 ( Pt 3), 427-438. doi:10.1348/000712600161916

Cvejic, E., Birch, R. C., \& Vollmer-Conna, U. (2016). Cognitive Dysfunction in Chronic Fatigue Syndrome: a Review of Recent Evidence. Current Rheumatology Reports, 18(5), 24. doi:10.1007/s11926-0160577-9

Cvejic, E., Lloyd, A. R., \& Vollmer-Conna, U. (2016). Neurocognitive improvements after best-practice intervention for chronic fatigue syndrome: Preliminary evidence of divergence between objective indices and subjective perceptions. Comprehensive Psychiatry, 66, 166-175. doi:10.1016/j.comppsych.2016.02.002

Delis, D. C., Freeland, J., Kramer, J. H., \& Kaplan, E. (1988). Integrating clinical assessment with cognitive neuroscience: construct validation of the California Verbal Learning Test. Journal of Consulting and Clinical Psychology, 56(1), 123-130.

Delis, D. C., Kaplan, E., \& Kramer, J. (2001). Delis-kaplan executive functions scale. In. San Antonio, TX: Psychological Corporation.

Delis, D. C., Kaplan, E., Kramer, J. H., \& Ober, B. A. (2000). California verbal learning test (CVLT-II) (2nd ed. ed.). San Antonio, TX: Psychological Corporation.

DeLuca, J., Christodoulou, C., Diamond, B. J., Rosenstein, E. D., Kramer, N., \& Natelson, B. H. (2004). Working memory deficits in chronic fatigue syndrome: Differentiating between speed and accuracy of information processing. Journal of the International Neuropsychological Society, 10(1), 101-109.

Denning, J. H. (2012). The efficiency and accuracy of the Test of Memory Malingering trial 1, errors on the first 10 items of the test of memory malingering, and five embedded measures in predicting invalid test performance. Archives of Clinical Neuropsychology, 27(4), 417-432. doi:10.1093/arclin/acs044

Devendorf, A. R., Jackson, C. T., Sunnquist, M., \& Jason, L. A. (2017). Defining and measuring recovery from myalgic encephalomyelitis and chronic fatigue syndrome: the physician perspective. Disability and Rehabilitation, 1-8. doi:10.1080/09638288.2017.1383518

Dickson, A., Toft, A., \& O'Carroll, R. E. (2009). Neuropsychological functioning, illness perception, mood and quality of life in chronic fatigue syndrome, autoimmune thyroid disease and healthy participants. Psychological Medicine, 39(9), 1567-1576. doi:10.1017/\$0033291708004960

Elkana, O., Falcofsky, A. K., Shorer, R., Bar-On Kalfon, T., \& Ablin, J. N. (2019). Does the cognitive index of the symptom severity scale evaluate cognition? Data from subjective and objective cognitive measures in fibromyalgia. Clinical and Experimental Rheumatology, 37 Suppl 116(1), 51-57.

Fredheim, O. M., Kaasa, S., Fayers, P., Saltnes, T., Jordhoy, M., \& Borchgrevink, P. C. (2008). Chronic nonmalignant pain patients report as poor health-related quality of life as palliative cancer patients. Acta Anaesthesiologica Scandinavica, 52(1), 143-148. doi:10.1111/j.1399-6576.2007.01524.x 
Friedberg, F., Dechene, L., McKenzie, M. J., 2nd, \& Fontanetta, R. (2000). Symptom patterns in longduration chronic fatigue syndrome. Journal of Psychosomatic Research, 48(1), 59-68. doi:10.1016/s0022-3999(99)00077-x

Fukuda, K., Straus, S. E., Hickie, I., Sharpe, M. C., Dobbins, J. G., \& Komaroff, A. (1994). The chronic fatigue syndrome: a comprehensive approach to its definition and study. International Chronic Fatigue Syndrome Study Group. Annals of Internal Medicine, 121(12), 953-959.

Goedendorp, M. M., van der Werf, S. P., Bleijenberg, G., Tummers, M., \& Knoop, H. (2013). Does neuropsychological test performance predict outcome of cognitive behavior therapy for Chronic Fatigue Syndrome and what is the role of underperformance? Journal of Psychosomatic Research, 75(3), 242-248. doi:10.1016/j.jpsychores.2013.07.011

Grafman, J., Schwartz, V., Dale, J. K., Scheffers, M., Houser, C., \& Straus, S. E. (1993). Analysis of neuropsychological functioning in patients with chronic fatigue syndrome. Journal of Neurology, Neurosurgery and Psychiatry, 56(6), 684-689. doi:10.1136/jnnp.56.6.684

Hughes, A., Hirsch, C., Chalder, T., \& Moss-Morris, R. (2016). Attentional and interpretive bias towards illness-related information in chronic fatigue syndrome: A systematic review. British Journal of Health Psychology, 21(4), 741-763. doi:10.1111/bjhp.12207

Ickmans, K., Meeus, M., De Kooning, M., Lambrecht, L., Pattyn, N., \& Nijs, J. (2015). Associations Between Cognitive Performance and Pain in Chronic Fatigue Syndrome: Comorbidity with Fibromyalgia Does Matter. Pain Physician, 18(5), E841-852.

Ickmans, K., Meeus, M., Kos, D., Clarys, P., Meersdom, G., Lambrecht, L., . . . Nijs, J. (2013). Cognitive performance is of clinical importance, but is unrelated to pain severity in women with chronic fatigue syndrome. Clinical Rheumatology, 32(10), 1475-1485. doi:10.1007/s10067-013-2308-1

Ingvar, M. (2015). Learning mechanisms in pain chronification--teachings from placebo research. Pain, 156 Suppl 1, S18-23. doi:10.1097/j.pain.0000000000000093

Jason, L. A., Richman, J. A., Rademaker, A. W., Jordan, K. M., Plioplys, A. V., Taylor, R. R., .. Plioplys, S. (1999). A community-based study of chronic fatigue syndrome. Archives of Internal Medicine, 159(18), 2129-2137. doi:DOI 10.1001/archinte.159.18.2129

Joyce, E., Blumenthal, S., \& Wessely, S. (1996). Memory, attention, and executive function in chronic fatigue syndrome. Journal of Neurology, Neurosurgery and Psychiatry, 60(5), 495-503. doi:10.1136/jnnp.60.5.495

Karsdorp, P. A., Geenen, R., \& Vlaeyen, J. W. (2014). Response inhibition predicts painful task duration and performance in healthy individuals performing a cold pressor task in a motivational context. European Journal of Pain (London, England), 18(1), 92-100. doi:10.1002/j.15322149.2013.00348.x

Klepstad, P., Loge, J. H., Borchgrevink, P. C., Mendoza, T. R., Cleeland, C. S., \& Kaasa, S. (2002). The Norwegian brief pain inventory questionnaire: translation and validation in cancer pain patients. Journal of Pain and Symptom Management, 24(5), 517-525. doi:10.1016/s0885-3924(02)005262

Krupp, L. B., Sliwinski, M., Masur, D. M., Friedberg, F., \& Coyle, P. K. (1994). Cognitive functioning and depression in patients with chronic fatigue syndrome and multiple sclerosis. Archives of Neurology, 51(7), 705-710.

Landrø, N. I., Celius, E. G., \& Sletvold, H. (2004). Depressive symptoms account for deficient information processing speed but not for impaired working memory in early phase multiple sclerosis (MS). Journal of the Neurological Sciences, 217(2), 211-216. doi:10.1016/j.jns.2003.10.012

Landrø, N. I., Fors, E. A., Vapenstad, L. L., Holthe, O., Stiles, T. C., \& Borchgrevink, P. C. (2013). The extent of neurocognitive dysfunction in a multidisciplinary pain centre population. Is there a relation between reported and tested neuropsychological functioning? Pain, 154(7), 972-977. doi:10.1016/j.pain.2013.01.013 
Lezak, M. D., Howieson, D. B., Bigler, E. D., \& Tranel, D. (2012). Neuropsychological assessment (5th ed. ed.). Oxford, UK: Oxford University Press.

Majer, M., Welberg, L. A., Capuron, L., Miller, A. H., Pagnoni, G., \& Reeves, W. C. (2008). Neuropsychological performance in persons with chronic fatigue syndrome: results from a population-based study. Psychosomatic Medicine, 70(7), 829-836. doi:10.1097/PSY.0b013e31817b9793

Marshall, P. S., Forstot, M., Callies, A., Peterson, P. K., \& Schenck, C. H. (1997). Cognitive slowing and working memory difficulties in chronic fatigue syndrome. Psychosomatic Medicine, 59(1), 58-66.

Mazza, S., Frot, M., \& Rey, A. E. (2018). A comprehensive literature review of chronic pain and memory. Progress in Neuro-Psychopharmacology and Biological Psychiatry, 87(Pt B), 183-192. doi:10.1016/j.pnpbp.2017.08.006

McDonald, E., Cope, H., \& David, A. (1993). Cognitive impairment in patients with chronic fatigue: a preliminary study. Journal of Neurology, Neurosurgery and Psychiatry, 56(7), 812-815. doi:10.1136/jnnp.56.7.812

Mitchell, A. J. (2008). Is it time to separate subjective cognitive complaints from the diagnosis of mild cognitive impairment? Age and Ageing, 37(5), 497-499. doi:10.1093/ageing/afn147

Moriarty, O., McGuire, B. E., \& Finn, D. P. (2011). The effect of pain on cognitive function: a review of clinical and preclinical research. Progress in Neurobiology, 93(3), 385-404. doi:10.1016/j.pneurobio.2011.01.002

Morriss, R. K., \& Wearden, A. J. (1998). Screening instruments for psychiatric morbidity in chronic fatigue syndrome. Journal of the Royal Society of Medicine, 91(7), 365-368.

Ojeda, B., Failde, I., Duenas, M., Salazar, A., \& Eccleston, C. (2016). Methods and Instruments to Evaluate Cognitive Function in Chronic Pain Patients: A Systematic Review. Pain Medicine, 17(8), 14651489. doi:10.1093/pm/pnv077

Pennington, C., Hayre, A., Newson, M., \& Coulthard, E. (2015). Functional Cognitive Disorder: A Common Cause of Subjective Cognitive Symptoms. Journal of Alzheimer's Disease, 48 Suppl 1, S19-24. doi:10.3233/JAD-150182

Pennington, C., Newson, M., Hayre, A., \& Coulthard, E. (2015). Functional cognitive disorder: what is it and what to do about it? Practical Neurology, 15(6), 436-444. doi:10.1136/practneurol-2015001127

Ray, C., Phillips, L., \& Weir, W. R. (1993). Quality of attention in chronic fatigue syndrome: subjective reports of everyday attention and cognitive difficulty, and performance on tasks of focused attention. British Journal of Clinical Psychology, 32 ( Pt 3), 357-364.

Royle, J., \& Lincoln, N. B. (2008). The Everyday Memory Questionnaire-revised: development of a 13item scale. Disability and Rehabilitation, 30(2), 114-121. doi:10.1080/09638280701223876

Santamarina-Perez, P., Eiroa-Orosa, F. J., Rodriguez-Urrutia, A., Qureshi, A., \& Alegre, J. (2014). Neuropsychological impairment in female patients with chronic fatigue syndrome: a preliminary study. Appl Neuropsychol Adult, 21(2), 120-127. doi:10.1080/09084282.2013.771264

Schmaling, K. B., \& Betterton, K. L. (2016). Neurocognitive complaints and functional status among patients with chronic fatigue syndrome and fibromyalgia. Quality of Life Research, 25(5), 12571263. doi:10.1007/s11136-015-1160-y

Shanks, L., Jason, L. A., Evans, M., \& Brown, A. (2013). Cognitive impairments associated with CFS and POTS. Frontiers in Physiology, 4, 113. doi:10.3389/fphys.2013.00113

Short, K., McCabe, M., \& Tooley, G. (2002). Cognitive functioning in chronic fatigue syndrome and the role of depression, anxiety, and fatigue. Journal of Psychosomatic Research, 52(6), 475-483. doi:10.1016/s0022-3999(02)00290-8

Siqveland, J., Sundseth, O. O., Dalsbo, T. K., Harboe, I., \& Leiknes, K. (2014). NIPH Systematic Reviews: Executive Summaries. In Psychometric Assessment of the Norwegian Version of the California 
Verbal Learning Test II (CVLT-II). Oslo, Norway: Knowledge Centre for the Health Services at The Norwegian Institute of Public Health (NIPH)

Copyright (c)2014 by The Norwegian Institute of Public Health (NIPH).

Strauss, E., Sherman, E. M. S., \& Spreen, O. (2006). compendium of neuropsychological tests: administration, norms, and commentary. In O. U. Press (Ed.). Oxford/New York.

Sullivan, K. A., \& Edmed, S. L. (2012). The good-old-days bias and post-concussion syndrome symptom reporting in a non-clinical sample. Brain Injury, 26(9), 1098-1104. doi:10.3109/02699052.2012.666367

Sunderland, A., Harris, J. E., \& Gleave, J. (1984). Memory failures in everyday life following severe head injury. Journal of Clinical Neuropsychology, 6(2), 127-142.

Teodoro, T., Edwards, M. J., \& Isaacs, J. D. (2018). A unifying theory for cognitive abnormalities in functional neurological disorders, fibromyalgia and chronic fatigue syndrome: systematic review. Journal of Neurology, Neurosurgery and Psychiatry. doi:10.1136/jnnp-2017-317823

Tesio, V., Torta, D. M., Colonna, F., Leombruni, P., Ghiggia, A., Fusaro, E., . . Castelli, L. (2015). Are fibromyalgia patients cognitively impaired? Objective and subjective neuropsychological evidence. Arthritis Care \& Research, 67(1), 143-150. doi:10.1002/acr.22403

Thomas, M., \& Smith, A. (2009). An investigation into the cognitive deficits associated with chronic fatigue syndrome. Open Neurology Journal, 3, 13-23. doi:10.2174/1874205X00903010013

Togo, F., Lange, G., Natelson, B. H., \& Quigley, K. S. (2015). Attention network test: assessment of cognitive function in chronic fatigue syndrome. Journal of Neuropsychology, 9(1), 1-9. doi:10.1111/jnp.12030

Van Den Eede, F., Moorkens, G., Hulstijn, W., Maas, Y., Schrijvers, D., Stevens, S. R., . . Sabbe, B. G. (2011). Psychomotor function and response inhibition in chronic fatigue syndrome. Psychiatry Research, 186(2-3), 367-372. doi:10.1016/j.psychres.2010.07.022

Vercoulen, J. H., Bazelmans, E., Swanink, C. M., Galama, J. M., Fennis, J. F., van der Meer, J. W., \& Bleijenberg, G. (1998). Evaluating neuropsychological impairment in chronic fatigue syndrome. Journal of Clinical and Experimental Neuropsychology, 20(2), 144-156. doi:10.1076/jcen.20.2.144.1160

Wearden, A., \& Appleby, L. (1996). Research on cognitive complaints and cognitive functioning in patients with chronic fatigue syndrome (CFS): What conclusions can we draw? Journal of Psychosomatic Research, 41(3), 197-211. doi:10.1016/0022-3999(96)00131-6

Wearden, A., \& Appleby, L. (1997). Cognitive performance and complaints of cognitive impairment in chronic fatigue syndrome (CFS). Psychological Medicine, 27(1), 81-90.

Wechsler, D. (2003). Wechsler adult intelligence scale [manual, norwegian version]. In (3rd ed. ed.). New York: The Psychological Corporation.

White, P. D., Goldsmith, K. A., Johnson, A. L., Potts, L., Walwyn, R., DeCesare, J. C., . . group, P. t. m. (2011). Comparison of adaptive pacing therapy, cognitive behaviour therapy, graded exercise therapy, and specialist medical care for chronic fatigue syndrome (PACE): a randomised trial. Lancet, 377(9768), 823-836. doi:10.1016/S0140-6736(11)60096-2

Zigmond, A. S., \& Snaith, R. P. (1983). The hospital anxiety and depression scale. Acta Psychiatrica Scandinavica, 67(6), 361-370. doi:10.1111/j.1600-0447.1983.tb09716.x 


\section{$\underline{\text { Tables }}$}

\section{Table 1}

Demographic, psychometric, and clinical characteristics in means and SDs for continuous variables and n (\%) for categorical variables

\begin{tabular}{|c|c|c|}
\hline Variable & Total $(n=236)$ & Range \\
\hline Age, years & $35.7(10.9)$ & $18-61$ \\
\hline Gender (\% female) & $194(82.2 \%)$ & - \\
\hline Education (\% BS/MS) & $75(31.8 \%)$ & High school - MS \\
\hline Fatigue (CFS) & $9.7(1.8)$ & $1-11$ \\
\hline Pain (BPI) & $4.3(2.1)$ & $0-8$ \\
\hline WAIS-III Matrix Reasoning & $12.5(2.6)$ & $5-18$ \\
\hline WAIS-III Vocabulary & $10.2(2.7)$ & $5-37$ \\
\hline EMQ & $4.3(1.3)$ & $1.3-8.4$ \\
\hline Depression (BDI-II) & $13.3(6.5)$ & $0-42.5$ \\
\hline Minimal 0-13(\% yes) & $132(55.9 \%)$ & - \\
\hline Mild 14-19(\% yes) & $55(22.3 \%)$ & - \\
\hline Moderate $20-28(\%$ yes $)$ & $44(18.6 \%)$ & - \\
\hline Severe $29-63(\%$ yes $)$ & $2(0.8 \%)$ & - \\
\hline Anxiety (HADS-A) & $6.8(3.9)$ & $0-18$ \\
\hline
\end{tabular}

BS/MS: Bachelor/Master degree; CFS: Chalder Fatigue Scale (0-33, where 33 is maximum); BPI: Brief Pain Inventory $(\mathrm{NRS} 0-10$, where 10 is maximum $)=($ mean last week + maximal + minimal pain $) / 3$; WAIS-III: Wechsler Adult Intelligence Scale-Third Edition (1-19, where 19 is maximum); EMQ: Everyday Memory Questionnaire (1-9, where 9 is maximum); BDI-II: Beck Depression Inventory-Second Edition (0-63, where 63 is the most severe; HADS-A: Hospital Anxiety and Depression Scale-Anxiety (0-21, where 21 is maximum). 


\section{Table 2}

Multiple linear regression analysis examining subjective cognitive function (EMQ) associated with objective neuropsychological tests with and without adjusting for fatigue, pain, depression and anxiety

\begin{tabular}{|c|c|c|}
\hline Variable & Unadjusted EMQ & Adjusted EMQ \# \\
\hline Stroop I & $\begin{array}{l}\beta=-0.108 \\
p=0.099 \\
\mathrm{R}^{2}=0.007\end{array}$ & $\begin{array}{l}\beta=-0.127 \\
p=0.077 \\
\mathrm{R}^{2}=0.001\end{array}$ \\
\hline Stroop II & $\begin{array}{l}\beta=-0.108 \\
p=0.099 \\
\mathrm{R}^{2}=0.007\end{array}$ & $\begin{array}{l}\beta=-0.035 \\
p=0.632 \\
\mathrm{R}^{2}=0.002\end{array}$ \\
\hline Stroop III & $\begin{array}{l}\beta=-0.117 \\
p=0.073 \\
\mathrm{R}^{2}=0.009\end{array}$ & $\begin{array}{l}\beta=-0.127 \\
p=0.083 \\
\mathrm{R}^{2}=0.018\end{array}$ \\
\hline Stroop IV & $\begin{array}{l}\beta=-0.163 \\
p=0.013 \\
\mathrm{R}^{2}=0.022\end{array}$ & $\begin{array}{l}\beta=-0.140 \\
p=0.056 \\
\mathrm{R}^{2}=0.026\end{array}$ \\
\hline CVLT-II learning & $\begin{array}{l}\beta=-0.162 \\
p=0.013 \\
\mathrm{R}^{2}=0.022\end{array}$ & $\begin{array}{l}\beta=-0.164 \\
p=0.026 \\
\mathrm{R}^{2}=0.015\end{array}$ \\
\hline CVLT-II delay & $\begin{array}{l}\beta=-0.165 \\
p=0.011 \\
\mathrm{R}^{2}=0.023\end{array}$ & $\begin{array}{l}\beta=-0.161 \\
p=0.028 \\
\mathrm{R}^{2}=0.011\end{array}$ \\
\hline WAIS-III L-N Sequencing & $\begin{array}{l}\beta=-0.167 \\
p=0.010 \\
\mathrm{R}^{2}=0.024\end{array}$ & $\begin{array}{c}\beta=-0.133 \\
p=0.069 \\
\mathrm{R}^{2}=0.040\end{array}$ \\
\hline PASAT & $\begin{array}{l}\beta=-0.121 \\
p=0.064 \\
\mathrm{R}^{2}=0.010\end{array}$ & $\begin{array}{l}\beta=-0.050 \\
p=0.483 \\
\mathrm{R}^{2}=0.018\end{array}$ \\
\hline
\end{tabular}

EMQ: Everyday Memory Questionnaire; Stroop I-IV from Delis-Kaplan Executive Functions System, ColorWord Interference test, CVLT-II: California Verbal Learning Test-Second edition; WAIS-III L-N sequencing: Wechsler Adult Intelligence Scale-Third Edition, Letter-Number Sequencing; PASAT: Paced Auditory Serial Addition Task. $\beta=$ standardized coefficients Beta; $\mathrm{R}^{2}$ : model adjusted $\mathrm{R}$ squared, coefficient of determination; * EMQ was adjusted with fatigue, pain, depression and anxiety. Note that standardized scores were used for the analyses. 


\section{Table 3}

Association between subjective health complaints (fatigue, pain, depression, and anxiety) and subjective and objective cognitive measures

\begin{tabular}{|c|c|c|c|c|}
\hline Variable & Fatigue & Pain & Depression & Anxiety \\
\hline EMQ & $\begin{array}{c}\mathrm{r}=0.363^{*} \\
p<0.001 \\
\mathrm{CI}=(0.233,0.476)\end{array}$ & $\begin{array}{c}0.249 * \\
<0.001 \\
(0.128,0.367)\end{array}$ & $\begin{array}{c}0.289^{*} \\
<0.001 \\
(0.163,0.396)\end{array}$ & $\begin{array}{c}0.037 \\
0.571 \\
(-0.096,0.170)\end{array}$ \\
\hline Stroop 1 & $\begin{array}{c}0.036 \\
0.578 \\
(-0.107,0.181)\end{array}$ & $\begin{array}{c}-0.022 \\
0.740 \\
(-0.145,0.094)\end{array}$ & $\begin{array}{c}-0.053 \\
0.422 \\
(-0.178,0.077)\end{array}$ & $\begin{array}{c}-0.012 \\
0.853 \\
(-0.143,0.107)\end{array}$ \\
\hline Stroop 2 & $\begin{array}{c}-0.182 \\
0.005 \\
(-0.351,0.115)\end{array}$ & $\begin{array}{c}-0.112 \\
0.085 \\
(-0.191,-0.004)\end{array}$ & $\begin{array}{c}-0.038 \\
0.565 \\
(-0.107,0.063)\end{array}$ & $\begin{array}{c}0.033 \\
0.617 \\
(-0.093,0.107)\end{array}$ \\
\hline Stroop 3 & $\begin{array}{c}0.038 \\
0.565 \\
(-0.108,0.168)\end{array}$ & $\begin{array}{c}-0.042 \\
0.522 \\
(-0.170,0.086)\end{array}$ & $\begin{array}{c}-0.081 \\
0.216 \\
(-0.206,0.043)\end{array}$ & $\begin{array}{c}0.035 \\
0.596 \\
(-0.099,0.168)\end{array}$ \\
\hline Stroop 4 & $\begin{array}{c}-0.059 \\
0.371 \\
(-0.190,0.084)\end{array}$ & $\begin{array}{c}-0.117 \\
0.075 \\
(-0.253,0.007)\end{array}$ & $\begin{array}{c}-0.054 \\
0.408 \\
(-0.175,0.064)\end{array}$ & $\begin{array}{c}0.093 \\
0.156 \\
(-0.038,0.222)\end{array}$ \\
\hline $\begin{array}{l}\text { CVLT-II } \\
\text { learning }\end{array}$ & $\begin{array}{c}-0.059 \\
0.364 \\
(-0.208,0.072)\end{array}$ & $\begin{array}{c}-0.103 \\
0.113 \\
(-0.224,0.040)\end{array}$ & $\begin{array}{c}-0.001 \\
0.983 \\
(-0.110,0.115)\end{array}$ & $\begin{array}{c}0.012 \\
0.860 \\
(-0.110,0.136)\end{array}$ \\
\hline CVLT-II delay & $\begin{array}{c}-0.025 \\
0.707 \\
(-0.157,0.106)\end{array}$ & $\begin{array}{c}-0.095 \\
0.144 \\
(-0.208,0.040)\end{array}$ & $\begin{array}{c}-0.066 \\
0.315 \\
(-0.172,0.047)\end{array}$ & $\begin{array}{c}0.024 \\
0.713 \\
(-0.093,0.143)\end{array}$ \\
\hline $\begin{array}{l}\text { WAIS-III L-N } \\
\text { Sequencing }\end{array}$ & $\begin{array}{c}-0.137 \\
0.035 \\
(-0.276,0.011)\end{array}$ & $\begin{array}{c}-0.121 \\
0.062 \\
(-0.253,0.006)\end{array}$ & $\begin{array}{c}-0.021 \\
0.751 \\
(-0.145,0.103)\end{array}$ & $\begin{array}{c}0.007 \\
0.914 \\
(-0.125,0.137)\end{array}$ \\
\hline PASAT & $\begin{array}{c}-0.121 \\
0.063 \\
(-0.248,0.009)\end{array}$ & $\begin{array}{c}-0.237^{*} \\
<0.001 \\
(-0.344,-0.124)\end{array}$ & $\begin{array}{c}-0.058 \\
0.373 \\
(-0.183,0.075)\end{array}$ & $\begin{array}{c}-0.007 \\
0.916 \\
(-0.133,0.126)\end{array}$ \\
\hline
\end{tabular}

EMQ: Everyday Memory Questionnaire; Stroop I-IV from Delis-Kaplan Executive Functions System, ColorWord Interference test; CVLT-II: California Verbal Learning Test-Second edition; WAIS-III L-N sequencing: Wechsler Adult Intelligence Scale-Third Edition, Letter-Number Sequencing; PASAT: Paced Auditory Serial Addition Task; r: Pearson correlation coefficient; CI: 95\% confidence interval; $*=p<0.001$. 


\section{Table 4}

Percentage of subjects $(\mathrm{n}=236)$ performing below 1.5 and 2.0 SDs from the normative mean $(\mathrm{z}$ scores) on the neuropsychological measures

\begin{tabular}{|l|c|c|}
\hline Neuropsychological variables & $<\mathbf{1 . 5}$ SD $(\boldsymbol{\%})$ & $<\mathbf{2 . 0}$ SD $(\boldsymbol{\%})$ \\
\hline Stroop test I & 38 & 20 \\
Stroop test II & 31 & 16 \\
Stroop test III & 21 & 11 \\
Stroop test IV & 28 & 16 \\
CVLT-II learning & 8 & 2 \\
CVLT-II delay & 6 & 4 \\
PASAT & 10 & 6 \\
WAIS-III L-N Sequencing & 7 & 1 \\
\hline
\end{tabular}

Stroop I-IV from Delis-Kaplan Executive Functions System, Color-Word Interference test; CVLT-II: California Verbal Learning Test-Second edition; PASAT: Paced Auditory Serial Addition Task; WAIS-III L-N sequencing: Wechsler Adult Intelligence Scale-Third Edition, Letter-Number Sequencing. 